\title{
Itinéraires
}

Itinéraires Littérature, textes, cultures

2010-4|2010

Modernités shakespeariennes

\section{Hugh Grady (ed.), Shakespeare and Modernity : Early Modern to Millenium}

Londres et New York, Routledge, 2000.

\section{Wendy Ribeyrol}

\section{(2) OpenEdition}

Journals

\section{Édition électronique}

URL : http://journals.openedition.org/itineraires/1763

DOI : 10.4000/itineraires. 1763

ISSN : 2427-920X

\section{Éditeur}

Pléiade

\section{Édition imprimée}

Date de publication : 1 décembre 2010

Pagination : 181-184

ISBN : 978-2-296-13183-5

ISSN : 2100-1340

\section{Référence électronique}

Wendy Ribeyrol, « Hugh Grady (ed.), Shakespeare and Modernity: Early Modern to Millenium », Itinéraires [En ligne], 2010-4 | 2010, mis en ligne le 01 décembre 2010, consulté le 22 septembre 2020. URL : http://journals.openedition.org/itineraires/1763 ; DOI : https://doi.org/10.4000/itineraires.1763

Ce document a été généré automatiquement le 22 septembre 2020.

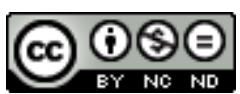

Itinéraires est mis à disposition selon les termes de la licence Creative Commons Attribution - Pas d'Utilisation Commerciale - Pas de Modification 4.0 International. 


\title{
Hugh Grady (ed.), Shakespeare and Modernity : Early Modern to Millenium
}

Londres et New York, Routledge, 2000.

\author{
Wendy Ribeyrol
}

\section{RÉFÉRENCE}

Hugh Grady (ed.), Shakespeare and Modernity : Early Modern to Millenium, Londres et New York, Routledge, 2000.

1 Shakespeare and Modernity: Early Modern to Millenium, publié au tournant du troisième millénaire est un recueil de neuf essais d'universitaires américains et britanniques, précédé d'une introduction de Hugh Grady, auteur par ailleurs de The Modernist Shakespeare (1991), Shakespeare's Universal Wolf (1996) et plus récemment de Shakespeare and Impure Aesthetics (2009). Ce livre offre à l'étudiant, à l'enseignant et au chercheur un état des lieux stimulant de l'évolution des études shakespeariennes depuis le début des années 1990.

2 Si le qualificatif de «moderne » revient fréquemment sous la plume des critiques de l'œuvre de Shakespeare, la raison en est, selon Grady, la capacité de celle-ci à soutenir des interprétations variées, parfois contradictoires, et sans cesse renouvelées. Chaque génération contemple et réinterprète les pièces du canon en les façonnant à partir d'un regard nouveau, «le présent aide à recréer le passé ${ }^{1}$ » écrira Terence Hawkes qui dirige la collection "Accents on Shakespeare" à laquelle ce volume appartient. À titre d'illustration, quoique quelque peu frivole, Linda Charnes dans son chapitre intitulé "The Hamlet formerly known as Prince», le dernier du recueil, explique que les frasques sexuelles du président américain William Jefferson Clinton peuvent colorer aujourd'hui notre vision d'Hamlet. Le protagoniste et son père défunt, obsédés tous deux par l'adultère, en arrivent à oublier une situation politique d'une importance autrement plus grave : la survie du pays. Le sort du royaume du Danemark, comme celui des États-Unis pendant les années 1980, dépend de la libido de son dirigeant. Plus 
sérieusement, il est évident que l'on ne peut plus regarder Le Marchand de Venise, dont il est beaucoup question dans ce recueil, en faisant abstraction de l'antisémitisme du $\mathrm{xx}^{\mathrm{e}}$ siècle. Un texte n'offre pas qu'un seul sens historiquement déterminé, il est capable continuellement d'en produire d'autres, selon le regard du lecteur. Terence Hawkes de nouveau: "Il est évident que nous devons lire Shakespeare d'un point de vue historique, mais dans la mesure où l'histoire est le résultat d'un dialogue sans fin entre le passé et le présent, comment pouvons-nous décider quelles circonstances historiques doivent être prioritaires dans ce processus, celles de Shakespeare ou bien les nôtres ${ }^{2}$ ?"

Dans un même ordre d'idées, Charles Whitney (" Ante-aesthetics. Towards a theory of early modern audience response ») analyse le rôle joué par les spectateurs dans la production du sens. Le théâtre public à l'aube de l'ère moderne était l'espace d'un échange de capital culturel, souligne l'auteur. Le spectateur n'était plus uniquement consommateur mais pouvait, selon sa propre expérience, recréer le produit qui lui était présenté. Cette réaction du public théâtral est un facteur clé dans les appropriations ultérieures de l'œuvre de Shakespeare.

Depuis la fin des années 1980, le terme early modern sert à décrire l'époque de Shakespeare. Il remplace d'autres termes couramment utilisés jusqu'alors, tels " élisabéthain », certainement plus précis, ou « Renaissance », porteur, sans doute, de plus de prestige selon Douglas Bruster. Il souligne dans son excellent essai intitulé "Shakespeare and the end of history ", que nous avons affaire à une étiquette vague. Il s'agit d'un outil pratique plutôt que d'une référence à une période historique bien précise. L'auteur affirme qu'early modern évoque un mouvement dans deux directions opposées : vers le passé - la période prémoderne, et vers l'ici et maintenant - l'ère moderne. La période ainsi qualifiée se situe donc à un tournant, à un moment de vastes changements politiques et socio-économiques, à une époque présentant un intérêt accru pour les questions de subjectivité, de nation, d'identité, de race et de genre (gender).

5 Particulièrement intéressants dans ce recueil sont les trois chapitres consacrés à la modernité du Marchand de Venise. John Drakakis («Jew. Shylock is my name») examine les préfixes aux répliques des personnages. Dans la version in-quarto de 1600 de la pièce, l'usurier de Venise est qualifié tantôt de "Iewe ", tantôt de "Shylock». Ce détail n'a pas jusqu'ici attiré l'attention des chercheurs. Anne Barton, par exemple, n'y attache que peu d'importance, déclarant que l'instabilité de ces préfixes résulte simplement de l'indécision de Shakespeare. Même s'il y existe des raisons matérielles, liées à l'imprimerie, qui pourraient expliquer l'interchangeabilité des appellations, pour John Drakakis, passer de «Iewe " à «Shylock» indique le passage du stéréotype vers un personnage individualisé, ce qui signale en partie la naissance de l'ère moderne. Le Juif démoniaque du Moyen Âge cède ainsi la place au financier dont les pratiques ont fondé le capitalisme moderne.

6 Les deux autres chapitres consacrés au Marchand de Venise de Lisa Freinkel («"Modern” anti-Semitism and the veil of allegory ») et d'Eric S. Mallin ("Jewish invader and the soul of state: The Merchant of Venice and science fiction ») s'attaquent à la question de l'antisémitisme. Freinkel insiste sur la coupure entre l'antisémitisme de l'époque prémoderne, fondé sur la religion, et l'autre que l'on pourrait qualifier de "moderne ", fondé sur la race. Celui-ci repose sur les théories raciales du $\mathrm{XIX}^{\mathrm{e}}$ siècle à une époque, où, ainsi que l'a montré J.-P. Vernant, «en même temps que se mettent en place les études savantes sur les Sémites et les Indo-Européens, on invente sous la figure 
imaginaire de l'Hébreu et de l'Aryen un couple providentiel qui, en livrant aux Occidentaux christianisés le secret de leur identité, leur confère leur titre de noblesse ${ }^{3}$ ... ». Cet antisémitisme moderne est distinct de la tradition médiévale qui informe la pièce de Shakespeare. Cependant, si comme l'explique l'auteur, les juifs sont définis par la religion, ils ne sont pas fondamentalement différents des chrétiens. Ils peuvent être "sauvés" par la conversion, tandis que s'ils sont définis par la race, une qualité immuable, ils ne peuvent être rachetés. A priori, Portia déguisée en Balthazar, le jeune avocat qui doit décider du sort d'Antonio, ne voit aucune différence entre Shylock et son débiteur, « Qui est ici le marchand ? Et qui le Juif? » (IV.i.170) et cette ressemblance représente une menace pour la communauté de Venise. Sur le même sujet, Eric S. Mallin évoque les films de science fiction où l'extraterrestre, l'alien, est facilement identifiable à cause de son aspect physique profondément différent du nôtre. L'ennemi, l'insupportable autre, est ainsi immédiatement reconnaissable. Cependant Shylock est le miroir, le Judenspiegel, dans lequel le chrétien se voit reflété. Le rôle de Portia lors de la scène du tribunal sera donc de démontrer publiquement la différence entre le chrétien et le juif pour pouvoir procéder à l'exclusion de ce dernier. Elle parvient à ses fins par le refus du sang, " [c]e billet ne te donne pas d'iota de sang» (IV.i.302) : le sang qui signifie le sacrifice du Christ dont le juif ne peut bénéficier, et le sang du chrétien auquel le non-chrétien ne doit, ni ne peut en droit attenter.

Le chapitre de Stephen Cohen intitulé «(Post)modern Elizabeth : Gender, politics and the emergence of modern subjectivity » montre que les périodes prémoderne, moderne et postmoderne sont toutes pertinentes dans le cadre d'un débat portant sur l'œuvre de Shakespeare. L'auteur démontre que la reine Élisabeth puisait tout à la fois dans le discours d'une époque révolue et dans le discours émergeant de l'époque moderne afin de se façonner une identité ambivalente et changeante en tant que souveraine à la tête d'une société profondément patriarcale. Cohen suggère que cette vision de l'identité, non plus fixe et immuable, mais trouble et insaisissable, peut être qualifiée de postmoderne. De sexe indéterminé, agissant parfois en homme, parfois en femme, la reine a, du moins partiellement, inspiré la création de certaines héroïnes des comédies de Shakespeare : Rosalind, Viola, Portia.

Peut-être cependant que la modernité de Shakespeare réside surtout, comme le suggère Douglas Bruster, dans son utilisation de la langue anglaise qui, au début du XVII siècle était, de manière reconnaissable, moderne. Bruster qualifie le premier in-folio de 1623 de «manuel du discours de la culture moderne » sans lequel les études de la période early modern n'existeraient certainement pas.

\section{NOTES}

1. Terence Hawkes, Shakespeare in the Present, Londres et New York, Routledge, 2002, p. 4.

2. Ibid., p. 5 .

3. J.-P. Vernant, préface à l'ouvrage de Maurice Olender, Les Langues du Paradis, Aryens et Sémites: un couple providentiel, Paris, Seuil, 1989, p. 11. 


\section{AUTEURS}

WENDY RIBEYROL

Université Paris-Est Créteil 